\title{
Duas traduções difíceis: transpor línguas e linguagens
}

\section{Two difficult translations: transposition of idioms and languages}

Luiz Fernando Ramos ${ }^{1}$ 


\section{Resumo}

Não se trata de um artigo acadêmico, mas de duas traduções de dramaturgias singularíssimas, do português para $\circ$ inglês e do inglês para o português, apresentadas por um pequeno ensaio que constextualiza os processos de traduação e encenação de cada uma das peças. Duas Páginas em Branco, de Qorpo-Santo, e Am I to Go, or, I'll Say So de Gertrude Stein, desafiam o tradutor na transposição das línguas e o encenador na transposição da linguagem literária à cênica.

Palavras-chave: Tradução; encenação; adaptação; cena; palavra

\section{Abstract}

It is not a paper, but two translations of very much singular dramaturgies, from the Portuguese to the English and from the English to the Portuguese, presented by a short essay which contextualize the processes of translation and staging of each one of the plays. Two White Sheets, from Qorpo-Santo, and Am I To Go, or, I'll Say So, from Gertrude Stein, challenge the translator in the transposition of languages and the metteur-en-scène in the transposition from page to stage.

Keywords: Translation; staging; adaptation; scene; word

E-ISSN: 2358.6958

Professor Titular do Departamento de Artes Cênicas da Escola de Comunicacões e Artes da Universidade de São Paulo (USP). Pesquisador do CNPq. Ifr@usp.br 


\section{Duas traduções difíceis: tranpor línguas e linguagens}

Não sendo um tradutor profissional, tenho experimentado nos últimos trinta anos algumas circunstâncias pedagógicas e investigativas que me levaram a empreender traduções desafiantes. Apresento aqui duas delas que têm em comum revelarem dramaturgos raros, pela forma singular como estruturaram seus "dramas", e exigirem ambas encenações inventivas.

Trata-se das experiências cruzadas, na lida com o português, minha língua natal, e o inglês, uma aptidão adquirida, de traduzir uma peça do brasileiro Qorpo-Santo (1829-1883) para o inglês e uma peça da norte-americana Gertrude Stein (18741946) para o português. As duas traduções aqui oferecidas de Duas Páginas em Branco (Two White Sheets) e de Am I To Go or I'll say So (Estarei para ir, ou, Vou dizer Isto) foram realizadas, a primeira, em meu doutorado sanduíche na Inglaterra, para montagem efetiva da peça em 1994, e a segunda, no exercício da docência, em 2003, para o trabalho de conclusão de curso de uma aluna. Em comum às duas traduções o fato de serem, nos dois casos, dramaturgias pouco convencionais que de algum modo alargam os limite da forma dramática e serem, ao mesmo tempo, difíceis para encenar. Na verdade, não tenho notícia de que qualquer um dos dois textos tenha sido jamais encenado, a não ser nas duas ocasiões aqui apontadas. Outro aspecto que aproxima os dois textos é sua impermeabilidade aos arranjos dramáticos habituais, o que por certo dificulta a tradução em um alinhamento narrativo lógico e indiscutível. Nos dois casos as tramas são possibilidades incertas que cada leitor/tradutor arrisca singularmente.

\section{Qorpo-Santo}

Minha leitura do teatro de Qorpo-Santo começou a se fazer, há quase trinta anos, quando iniciava meu doutorado. Uma primeira tentativa de aproximação tratou de aplicar o modelo analítico desenvolvido por Peter Szondi em Teoria do drama moderno (Szondi, 1987), que dava conta da produção dos dramaturgos que precipitaram rupturas na forma do drama na virada dos séculos 19 para o 20. A tese central de Szondi, sem separar forma e conteúdo e pensando-os dialeticamente, percebe os dramas modernos como resultantes dos novos temas que, a partir do final do século 19, passaram a forçar a forma dramática a modificar-se para acolhe-los. Assim, o que ele chamou para efeito de raciocínio de "drama absoluto", estabelecido na Renascença e que teria apenas o diálogo entre os personagens como suporte para a exposição temática, começaria a se desestabilizar desde Ibsen, por exemplo, quando temáticas da subjetividade, como a memória reprimida de fatos passados ou pulsões de desejos inconscientes, exigiriam novas soluções formais. Como demonstrou Szondi, no caso de Ibsen, um exímio praticante da "peça bem feita", esta pressão de conteúdos subjetivos sobre a forma dialógica não chegava a fraturar o drama, mas deixava-o pleno de tensões. Em Strindberg a subjetividade já se impôs como eixo da ação e o dialogismo, ainda que presente, tornou-se subalterno. A tensão entre esta voz unilateral e a forma dramática provocaria rachaduras e faria emergir uma nova 
forma - o "drama de estações". Em Tchecov, sob o signo da resignação, as personagens já renunciavam ao presente, tempo por excelência do drama, e se intoxicavam de memórias e esperanças vãs. Esvaziados da função de carregar a ação os diálogos tornavam-se pretextos, paisagens ocasionais a esconder a inapetência em agir das personagens. A forma dramática tornava-se um disfarce patético ao dilaceramento das subjetividades.

O trabalho de Szondi, como é bem sabido, avança pelo século XX mostrando como os dramaturgos mais relevantes foram respondendo cada vez mais radicalmente às exigências das novas temáticas, sobretudo as sociais, até a formalização acabada do Teatro Épico. Na minha perspectiva, que buscava um diálogo entre a isolada e extemporânea dramaturgia de Qorpo Santo e a lógica historicista proposta por Szondi, fui encontrar numa dramaturgia européia também pouco conhecida um paralelo possível. Trata-se da pequena, mas significativa dramaturgia do poeta do expressionismo alemão Gottfried Benn, que entre 1914 e 1917 escreveu quatro peças. David Graver demonstrou como, a partir de uma visão absolutamente crítica da realidade, Benn questionou em seu teatro a própria constituição ontológica do real, que se lhe aparecia com uma desoladora inconsistência. Segundo Graver (1995), Benn construiu uma obra dramática que abarcou a incerteza e o caos, antes de estabelecer uma ordem momentânea. Benn tentaria retratar a desintegração do ego, confrontado-o às outras consciências e ao mundo. Para compreender este ceticismo extremamente epistemológico, como o definiu Graver, seria preciso reconhecer que ele se sustentava no solipsismo insistentemente cultuado por Benn (Graver, 1995; p. $x i+253$ ). Ao afirmar a existência de um sujeito autoconsciente a ponto de estabelecer-se completamente autônomo da realidade, Benn criaria um abrigo para o vazio e a vacuidade a que seu nihilismo tinha reduzido o mundo exterior. Tudo que era externo ao sujeito ameaçava sua integridade, convertendo-se em fantasmagoria na consciência individual. Guardadas as proporções entre este ceticismo radical do expressionismo alemão em plena primeira guerra mundial, e a situação de isolamento psíquico e social de Qorpo Santo às margens da Guerra do Paraguai, pareceu-me que a chave solipsista seria produtiva na análise das peças qorposantenses. Outra aproximação feita na época, quando o enfoque ainda buscava um diálogo com a leitura de Szondi do drama moderno, foi com a dramaturgia do futurismo italiano, cujas "Sínteses" - peças curtíssimas - traduzi para o português e encenei em 1985. Na sua economia e na sua estudada ruptura de sentido a dramaturgia futurista buscava desesperadamente chegar aonde Qorpo Santo, sem um programa explícito e provavelmente à custa de muito sofrimento mental, tinha alcançado com uma certa facilidade. De fato, pode-se dizer que em Qorpo-Santo não chega a haver um drama enquanto forma acabada. Há sim um quase drama, que como uma fumaça densa se esboça como figuração, mas logo se dissipa antes que se possa fixá-la. Nesse sentido a tensão entre forma e conteúdo, entre um discurso subjetivo esquizofrênico e uma forma dramática rala na sua determinação, de tão aguda, quase inviabiliza o drama no sentido conceituado por Szondi, o que não quererá dizer que inviabilize o teatro em si.

Esta linha de reflexão ganhou uma nova perspectiva quando, já no meu proces- 
so de doutoramento, passei a trabalhar as relações e contradições entre o literário e o cênico no processo teatral. Para localizar estas tensões, e contornar a complexidade metodológica da semiótica do espetáculo, optei por trabalhar verticalmente em torno das rubricas. Neste enfoque, voltado sobretudo para os aspectos cênicos, a dramaturgia de Qorpo-Santo ganhava novos ares e sugeria uma leitura para além dos aspectos literários, que sempre tinham prevalecido em análises precedentes. A colaborar neste entendimento mais amplo de sua obra dramática, o próprio Qorpo-Santo escreveu a seguinte nota final, na última página do seu conjunto de peças:

As pessoas que comprarem e quiserem levar à cena qualquer das Minhas Comédias - podem; bem como fazerem algumas alterações, corrigir alguns erros, e algumas faltas, quer de composição, quer de impressão, que a mim por inumerosos (sic) estorvos - foi impossível. (Qorpo-Santo, 1980, p. 394)

A seu jeito amalucado, e numa sinceridade constrangedora, ele estava antecipando um procedimento que se tornaria habitual no teatro, a partir dos anos sessenta do século passado, das encenações produzirem novos textos a partir dos originais gerados pelos dramaturgos, textos que, como antecipou Raymond Williams, genialmente, em Drama em cena, tenderiam a se tornar cada vez mais "scripts, histórias que os outros adaptavam para a cena" (Williams, 2010, p.229). Williams acrescentará na sequência que o escritor diretor, ou, mais frequentemente o "diretor-escritor", emergiria como a figura dominante nas décadas seguintes. Pois bem, tendo realizado esta investigação em profundidade sobre a rubrica, que não cabe aqui detalhar, reli a obra de Qorpo-Santo constatando como suas rubricas revelavam uma implícita e surpreendente intuição de teatralidade (ao que se saiba sua única experiência com teatro remeteria a um grêmio estudantil na época de adolescência, ao que não há nenhuma menção na Enciclopédia) e como, por meio desta era possível estabelecer uma ponte concreta com a contemporaneidade, mais até do que por uma leitura psicológica ou temática. A presumível potencialidade teatral foi testada quando encenei uma daquelas peças num país e numa língua estrangeiros, nas circunstâncias excepcionais de uma residência no Departamento de Teatro do Royal Holloway College, da Universidade de Londres. Traduzir Qorpo Santo para o inglês foi um desafio à parte, mas apresentá-lo cenicamente a um público pouco habituado a tais inconsistências dramáticas foi mais desafiador ainda. O resultado mais expressivo desta experiência foi comprovar a amplitude cênica de Qorpo-Santo, e isto a partir de uma de suas peças menos estruturadas e mais intrigantes, até por ser uma das mais inconsistentes dramaticamente. Trata-se de Duas Páginas em Branco, traduzida como Two White Sheets. A peça foi apresentada numa curta temporada no Teatro Studio do Royal Holloway College, em maio de 1994. Cabe esclarecer que o princípio construtivo desta montagem foi determinar todos os pontos de ruptura da ação dramática, que chegam a ser doze, cinco no primeiro e sete no segundo ato. Por pontos de ruptura eu entendo as guinadas radicais contabilizáveis no desenvolvimento da trama, tais como as modificações abruptas na trajetória dos personagens, ou mesmo sua desaparição ou mudança de nome, sem que qualquer necessidade dramática ou narrativa se apresentasse. Para demarcar esta pontuação no próprio espetáculo lancei mão 
de um recurso só justificável naquela circunstância cultural e social. Explicando melhor, todas as vezes que tais guinadas ocorriam o público escutava o som que estava habituado a ouvir nas estações do metrô londrino, quando os trens paravam nas estações. É o "Mind the Gap" pronunciado por uma voz gravada, de modo bem lento e afetado, para que os passageiros tenham consciência do vão entre os trens e as plataformas. Deslocado para a cena, aquele ruído familiar tratava de atentar o público para as bruscas reviravoltas que a cena lhe proporcionava.

A cena em Qorpo Santo é particularmente interessante porque as rubricas que a projetam exigem uma opção de linguagem cênica contundente, mais incisiva do que qualquer diálogo. Para exemplificar isto vale citar um trecho curto do início do segundo ato da peça, numa cena que fulgura entre as tantas preciosidades encontráveis naquela dramaturgia. Para se avaliar melhor o impacto da cena, vale retroceder ao primeiro ato. Ali a peça começava em uma casa de família com um dito professor ensinando a duas jovens alunas suas devidas lições. Durante este primeiro ato ocorrem várias mudanças bruscas de tom e rumo, que variam do brejeiro ao sensual e até ao quase erótico. Mas a ruptura decisiva ocorrerá nesse início do segundo ato quando, passando uma borracha sobre tudo que havia acontecido antes, Qorpo-Santo dispõe no espaço da suposta casa de família duas mesas de bilhar, com o requinte de colocar um jogador jogando solitariamente em cada uma delas. Esta imagem que lancina o isolamento solipsista, será logo interrompida pelo mesmo casal do início. Mas se antes os dois figuravam o professor sisudo e a aluna tenra e ingênua, agora aparecem casados, e dados a licenças de uma vida sexual despudorada.

\author{
Ato Segundo - Cena Primeira \\ Dois bilhares no cenário, cada um com o seu jogador - Pedro e Paulo.
}

Pedro (depois de algumas tacadas) - Não há melhor modo de jogar! Trabalho há duas horas para fazer uma carambola! E ainda o parceiro se não incomoda! Que diz amigo? (e continuando sempre) não é assim ?

Paulo - Sem dúvida! Eu também me tenho divertido à grande, e o que é melhor é que nada se perde.

Pedro - Oh! Isso é nada! Os jogadores se não incomodarem é que...que acho muito mais apreciável! (salta uma bola.) Se pilha algum passante, era capaz de lhe furar a barriga! Que diz, amigo Paulo?

Paulo - É verdade; somos parceiros sem parceiros (salta outra bola.) Oh! Foi bola rara! Mas o que vale é que encontrou uma forte parede!: mais que as muralhas de Sebastopol; não comparo com o Humaitá porque me parece inferior àquelas! Estou cansado (atirando com o taco! Saem de um lado os novos esposos.). (Qorpo-Santo, 1980, p.361)

Foi um desafio transpor essa dramaturgia singular para o inglês, não menor do que o de fazer os atores que colaboraram na montagem, formados na melhor tradição stanislavskiana inglesa, de construção psicológica dos personagens, desempenharem na errância e inconsistência dramática qorpo-santense. Acrescente-se que além do texto de Duas Páginas e Brancos, a montagem utilizou como prólogo um trecho de $R e$ lações Naturais, a peça mais conhecida de Qorpo Santo. A tradução aqui apresentada acrescenta esse adendo, seguindo o texto que foi efetivamente encenado. 


\section{Gertrude Stein}

Uma outra experiência de tradução com dramaturgia pouco convencional ocorreu com a obra dramática da escritora norte-americana Gertrude Stein (18741946). Ela tem em comum com Qorpo Santo o fato de ter criado obras dramáticas que não encontraram ecos imediatos na cena, e só foram acolhidas no palco muito tempo depois de criadas. No caso de Am I Go or I'll Say So, não houve propriamente uma montagem. A aluna que me solicitou a tradução, para realizar o seu projeto final de direção no curso de Artes Cênicas da ECA/USP, acabou fazendo um uso lateral da peça na performance instalação que montou em uma das salas do Departamento de Artes Cênicas. Por certo essa opção era respeitável, já que o texto, como se verá, não cumpre definitivamente os protocolos de um mecanismo dramático facilmente acionável na cena. Como uma dramaturgia que resiste à encenação, Estarei Para Ir, ou, Vou Isto Dizer está aberta a infinitas possibilidades cênicas, inclusive a que restou "encenada" ou não encenada. Como aponta James R Mellow, no prefácio do volume que reúne a maioria de suas peças - Operas and Plays -, publicado a primeira vez pela própria Stein, em 1932, e reunindo textos escritos entre 1913 e 1931,

Estes exercícios teatrais - que começam, ela nos conta, com $O$ Que Aconteceu: Uma Peça; uma peça em que nada acontece - logo desenvolveu-se em uma dramaturgia esvaziada completamente dos elementos essenciais: trama, desenvolvimento de personagens, cenário, rubricas. Mesmo a estrutura dramática, se pode ser chamada assim, não distingue entre cenas e atos. O que restou foi o ator (nem sempre identificável) e a palavra escrita. [...] Elas são literalmente - e literariamente - "peças de palavras", clara evidência da intenção de Stein de fazer da palavra o elemento prevalente de sua investida teatral. (Mellow , 1987, p.7) ${ }^{2}$

No Brasil a obra dramática de Gertrude Stein ainda é pouco conhecida e menos ainda encenada. Às iniciativas pioneiras de tradução de sua prosa, como as de Augusto de Campos, nos anos 1980 (Stein, 1989), seguiram-se outras tentativas, com destaque para o trabalho fundamental de Dirce Waltrick do Amarante e Luci Collin (Stein, 2014), que traduziram para o português dezoito das peças escritas entre 1913 e 1920, e das não menos relevantes colaborações de Júlio Castagnõn Guimarães, que traduziu cinco peças de Stein (Stein, 2000), de Inês Cardoso (Moreira, 2005) que traduziu quatro (Stein, 2001, 2005, 2010), e de Fábio Fonseca de Melo (s/d.), que traduziu uma. Com a tradução de $A m$ I to go, or, I'll say so aqui oferecida, tenciono me inserir nesse esforço coletivo de divulgacão dessa obra tão singular quanto mal assimilada, sobretudo no teatro brasileiro.

2 Ver também Ryan, 1984. 


\title{
Estarei para Ir ou Vou Isto Dizer
}

\author{
Gertrude Stein \\ Tradução Luiz Fernando Ramos ${ }^{3}$
}

Um curioso exemplo de como não é só com estrangeiros mas com seus concidadãos que eles acontecem, de fato eles acontecem com até mais sucesso e melhor com seus próprios concidadãos, com seu próprio concidadão.

\section{Uma Peça Em Lugares}

Perto Annency, Paris, Vence, Tahors, Alguns mais.

\section{Em primeiro lugar}

Objetos numa mesa e um levantamento de pontes e estradas.

Interlúdio. Em geral, O general gosta de seu café frio.

Interlúdio. A guerra.

Interlúdio. Não a guerra.

Em segundo lugar.

Paris. Como ele ocupam seus quartos. De um jeito. Eles dizem que algum tempo no dia, um dia inteiro. Um dia inteiro todo dia. Duas vezes ao dia. Dois dias, Pelo menos dois dias por semana. Pelo menos dois. Não como se poderia esperar um incidente, qualquer tal incidente. E um uso e um uso para aquilo.

No terceiro lugar.

Vence. Corretamente na estrada para Vence e uma vez lá, será que vamos admitir que Shakespeare era como aquilo fosse imodesto, imodestamente adquirido. Será que vamos admitir que não há gota e gotejar de novo gotejá-lo de novo. Ele dirá isto. Em quarto lugar.

Tahors, e muito muito mais, entre e sozinho, ele sozinho procura evitar não evitar, desertar não desertar, contratar não contratar, indicar não indicar, reclamar não reclamar, compartilhar não compartilhar estranhar e trocar muitos para mais e finalmente e muito rápido para trocar muito mais não trocar e ao longo para esperar para esperar recusar indicações. No cômputo final tudo participa e ele será que desiste de seu quarto.

Em quinto lugar.

Um senador, um senador é recebido concretamente ele é obrigado a recuar da cidade de seu coração e de seus sonhos e ele é impelido a colaborar na sustentacão de um pleito, ele não pleiteia si próprio mas ele é o único apoio e portanto não é obrigação, ele não tem satisfação nem retrato e nem iniciativa, ele tem tem ele tem sido gentileza ela própria em seu próprio interesse e no interesse ele sente. De fato ele dirá isso e diz e quer ser substituído, quer ser substituído e não pode se ajudar exatamente.

3 Texto - fonte: Gertrude Stein, Opera \& Plays, ed. James Mellow, New York, Station Hill Press, 1987. 
Nesse meio tempo e se fosse uma estação diferente do ano ali seria sol radioso. A cortina levanta-se no começo do domingo. É como se fosse o clima habitual.

\section{No primeiro lugar.}

Objetos numa mesa e um levantamento de pontes e estradas.

Toda uma cena em uma questão. Era ele ou era ele e seu irmão e era ele e mensagens e era ele era sua tia era sua mãe era ele e eram ele e seu irmão, eram eles, era ele, era ele uma maravilha era ele, não chegou ele com o trem onde for que ele tenha estado longe. Tivesse ele estado longe todo esse tempo. Fosse ele inexterminável, era ele e terá ele o declínio das montanhas. Montanhas começam a declinar, será ele erradicável declinarão as montanhas, será ele e será: e montanhas e decliná-las.

\section{Uma conversação com a família.}

Ele e o irmão dele e a irmã dele e o marido dela e seu pai e se ele tem e sua mãe e sua irmã e eles têm e filhos dela e eles significam mais.

Quando você olhar isso se lembre de mim.

Conversações não montadas atropeladamente.

Somava para isso.

Conversações.

A irmã. Encanando.

O pai. Endereçando.

O irmão. Vencendo.

A mãe. Sem auto-abnegação.

O cunhado. Não foi bem sucedido quando como eles poderiam dizer, ele pode dizer isto.

$\mathrm{Na}$ verdade à medida que ele será ele não realmente será ameaça ao segredo.

Conversações quase o tempo todo e ele já está praticamente lá.

$$
\text { primeiro interlúdio. }
$$

\section{Cena.}

Mimada de leve.

Por que ele todo enquanto porque ele como se fosse justamente arregalado.

Não é comodidade e para argumentar não alimenta perseverança. Perseverança para ser alimentada. Nesse caminho trocas são extemporâneas.

Uma conversação entre eles.

Ativamente rapidamente.

Resposta.

Rapidamente e ativamente.

Nem quando.

Resposta.

Agora e então. 
Ativamente e mais rapidamente.

Resposta.

E quando não mais rapidamente.

Agora e então mais ativamente.

Resposta.

Agora e então não mais que ativamente e rapidamente.

Mudança.

Mudança de administração.

Em geral.

Depois que o uso dos mimeógrafos foi introduzido.

Tudo foi muito bem tanto ou.

Ou mais.

Conversações com um general.

Se eles deixam para eles.

Não naquele caso.

Ele perguntou a ele diretamente.

E escolhido.

Ele escolheu deixar seu chapéu e bengala como se isso fosse em um domingo.

Regularmente falando ele falou para si próprio.

Em resposta.

$E$ isso é o que eles disseram.

Se eu não tivesse vindo.

E vem.

Eu venho.

Não encontrares gerais.

Em um general.

Isso em um geral

Aquilo em um general.

Para um general.

Antes do geral.

Nesse caso não em general.

Não gozar.

Não irritar.

Não general.

E em encontrando.

Se tivesse encontrado.

Polidamente.

Engendrando e copiando e tinta e aceitando e todo dia tem seu tem um dia.

Tivesse você um dia.

Tenha você tido um dia.

Se você me acreditar eu fui embora.

Nesse caminho melhor e melhor nesse caminho e melhor e melhor. 
Não significar dois anos exceto para o dia.

Eu aceito para hoje.

Não ser excedido em generosidade.

Escreveu ele de novo algum dia.

Não de novo.

E não de novo.

Então vem ou então vem ou então e então e como para eles.

Nenhuma divisão pode saber que dividir é fazer divisão.

O general gosta.

Se ele gosta.

Se ele gosta de ser avisado

E se ele gosta disso ser avisado

E se ele gosta disso igualmente.

E se ele avisa igualmente.

E se avisar.

E como se contar.

Muito bem.

Esse fim de tarde se necessário.

E assim administração estava no recital.

Pra recontar.

Não para recontar como homens e lugares.

Ele era de grande importância e no futuro isso poderia ter estado no futuro.

E agora apresentar armas.

Congratulações.

Não cadeiras.

Não não cerejas.

Porque faz isso porque como você faz.

Ou.

Para a porta.

Comece agora.

Começar agora.

Isso é o que eles disseram. O outro dia.

Interlúdio

A guerra

Por que o papel é escasso.

O papel não é muito escasso.

Interlúdio

Não a guerra.

Ou não a guerra.

De fato ou não a guerra. 
Ou de fato ou não a guerra.

Papel de desenho não é escasso.

Ou no papel de desenho o papel de desenho não é escasso;

Nem o papel de desenho é escasso.

Ou em ou não na guerra.

Tanto ou.

Ou a guerra.

Ou não a guerra.

Ou papel de desenho.

Ou papel escasso.

Ou papel não é escasso.

Ou papel de desenho.

Ou naquele caso.

ângulos razoavelmente parelhos.

Ou mesmo tão razoavelmente como para ângulos.

Ou ser como ser.

Como é ser como uma aptidão.

Como uma aptidão para Julho.

Em segundo,

Está acontecendo pra ele está acontecendo nele, está rapidamente tornando-se em origem como origem, está rapidamente acontecendo nele, está rapidamente acontecendo pra ele está rapidamente tornando-se como na origem.

Não na conversa.

Um quadro.

Enquadrar.

Como um quadro.

Como se num quadro.

Culpar.

Não houve exame sobre o qual jogar a culpa. Houve o exame e houve um quadro e não era para culpar. Não foi vergonha vergonha sentida vergonha ninguém ninguém saberá o nome. E dá na mesma ele era para culpar e culpar como a culpa de circunstância como a circunstância se parece como para o mesmo. O mesmo. Tudo na mesma. Tudo na mesma frente a essa circunstância não era para culpar nem como à menção de uma moldura. Nem uma mudança de idéia. Pense, você pensa, você se importa. Sim você se importa.

Toda a mudança.

Toda a mudança era era que objetos não estivessem sobre a mesa mas no hall e então conversas no quarto foram transferidas para estarem como se numa praça.

Uma praça pode ser uma pintura, uma porta ou nem tanto assim. Nem tanto assim embolorada.

Embolorada mundo estrangeiro.

Introdução para alcançar. Alcançar e rico conte-me que mico. 
Nesse caminho os começos começam.

Começam agora.

Começar.

Começo e começado.

No sul não há sol e nas montanhas não revólver. Nenhum revólver.

\section{Começado}

Começa de novo.

Em terceiro lugar.

Variedades são como para óleos e sapatos. Variedades como escolher como escolher variedade, como para variar, mais verões, mais como para verões e mais e mais para e como para mais verões.

Invernos como para invernos como para mais e mais, mais e mais como para invernos.

Como para verões e como para invernos mais como para verões e mais e mais como para invernos.

Como para perfeitamente bem, muito bem, como para muito bem, muito bem e muito muito bem, como para muito bem, como para muito muito bem, como para muito bem, como para muito muito bem. Como para como contar como se como contar para e muito bem como se como para contar muito bem. Como se para e muitíssimo mais. Como se para. Não como compromisso.

Em quarto lugar.

Entre.

Entre não importa o que Nelly tinha tido.

Um. Entronizado.

Dois. Entronizar.

Três. Palácio.

Quatro. Ministros.

Quatro. Ampulheta.

Seis. Pratos.

Sete. Tickets.

E oito. Um oito.

Por entre.

Destronar.

Dois. Ampulheta

Três. Fastio.

Cinco. Expeli-lo

Quatro. Não mais.

Dois. Acerca do corpo.

Um. Um exemplo.

Por entre.

Mensurando.

Dois. Não mensurando. 
Três. Peça isso de qualquer jeito.

Quatro.Retratos.

Quatro retratos de lona e não laço.

Não laço.

Não por entre

Não tela.

Não não.

E não.

Não nem por isso.

Uma questão. Qual é a diferença entre Waldemar e Jorge.

Resposta. Não há nenhuma.

Não no intervalo deles.

Ele encontra com ele foi encontrado com e pelo.

Compra.

Por e por.

Compra e não por e por.

Não por e por.

Não comprar e compra.

Não comprar e compra.

Não por e por.

Por e por.

Quem disse.

Ele disse.

Tema com variações.

Em quarto lugar.

Tahors e clímax.

Sem clímax.

Não como para um clímax.

Em quarto lugar e Tahors e mais.

Mais e Tahors.

Mais.

Em quarto lugar.

E mais.

Era um cumprimento como era como um cumprimento, era como era para ser como ser cumprimento, em quarto lugar. Um cumprimento em quarto lugar.

Favorecer em quarto lugar.

Como um favor em quarto lugar e como o quarto lugar como para o quarto lugar.

Quatro lugares.

Em todos os quatro lugares.

Como para todos os quatro lugares.

Em quarto lugar.

Como para o quarto lugar.

Era como para o quarto lugar.

Um lugar e não no lugar. 
E não como não colocar.

No quarto lugar.

Não em quarto lugar.

Em quarto lugar.

Tão cedo, qualquer um dele ou dela o mais cedo, tão cedo.

Em um lugar.

Pode colocar.

Eles têm um lugar.

Ele pode colocar.

E pode colocar.

Sem lugar.

Um lugar.

Lugar.

Colocar.

Em um lugar.

Em quinto lugar.

Quarto lugar.

Um quarto lugar.

Em quarto lugar.

No lugar.

Naquele lugar.

Colocar colocar um lugar meu lugar, meu lugar tu lugar nós lugar Eu lugar Eu lugar tu lugar, nós lugar, meu lugar, meu lugar, porque lugar, tu lugar meu lugar Eu lugar meu lugar, Eles colocaram um lugar, por um lugar como por um lugar, para e como pelo lugar, nos seus lugares em meu lugar. Eu e meu, colocar e meu lugar.

Em quarto lugar.

Por que.

Por que em quinto lugar.

E por que em quarto lugar.

E eu e meu lugar.

E eu.

E meu lugar.

E meu lugar.

E eu.

Em quarto e quinto lugares.

Pr'amanhã e não pr'amanhã, pra manha e não pra manha, pra emprestar e pra não emprestar, oh queridinha eu não. E então.

Pr'amanhã e não pr'amanhã ' pra manha e não pra manha para emprestar e não para emprestar, oh queridinha eu não.

E assim pão conhecido como salsicha, couve flor conhecida como ovo, manteiga conhecida como laranja e assim salada conhecida como salada salada crescida e conhecida e ovos dispostos e pesados e alcachofras escolhidas e congeladas e cevada plantada e crescida e trigo cultivado e colhido e cerejas mendigadas e agradecidas, não para ser agradecida por não ser agradecida por e retribuindo e como um presen- 
te. E como um presente. Cumprimentos e sorrir um instante, sorrir e cumprimentar e agora tudo muito bem e agora, pretender não fingir para e por isso e antes antes disso e ao lado ao lado mais, e mais não qualquer mais e a sorte não como a sorte, encontrar não na ou pela rua não pela rua, você compete, você o faz, e você e você alimenta você alimenta afinal você afinal alimenta-se disso afinal. Você come muito. Você come muitíssimo. E sempre como se pode dizer pato selvagem abaixo. Para de repente salvar tanto e muito mais. Muito e tanto mais e assim lá. Tudo certo e assim lá. Acontece de você saber o endereço de qualquer um de qualquer um e qualquer um. Mr. Mansard não é desprezível.

\section{Two White Sheets}

Qorpo-Santo
Translation Luiz Fernando Ramos
CHARACTERS:
Professor Smart
Mancília
Esterquilínia
Rocalipsa
Isolina
Peter
Paul
The soldier
A policeman
A lieutenant
Soldiers

PROLOGUE (From first scene of "Natural Relations") ${ }^{5}$

Professor Smart - I was thinking of going out; Going to see someone; but since my ungrateful and disgusting imagination robbed me of a dinner, I will try at least to talk with the one who offered it to me...However, I'm not sure that I will. In fact, I don't know what inspired me to carry on with such unrewarding work! I'll get up; continue it; and, perhaps, write on a dead man: perhaps on this one for whom, now, the echoes which inspired tears and pain awake. Arise in the hearts of those who hear the prayier for the soul of that one, whose days God has finished with his omnipotent voice or wish!

4 QORPO-SANTO, José J.C.L, Teatro Completo. Rio de Janeiro: MEC/SMT/FUNARTE, 1980.

5 Aqui se recortou um trecho da primeira cena da peça "Relacões Naturais", in Qorpo Santo, op.cit. p.65, como forma de introduzir melhor o autor e suas características idiosincráticas ao público inglês. 
(stands himilself up; gets closer to the table; takes a pen; wets with ink; and starts to write)

Prof. Smart - Today is the fourteenth of may, 1866. I live in the city of Porto Alegre, capital of the Province of Saint Peters of the South, and for many - Brazil's Empire... You can already see that this is a true comedy.

(throwing away the pen, and shouting)

Prof. Smart - To hell with this writer's life! It is better to be a comedian, a clown, a fool, or a director! I have just been writing, and writing, and writing; and never reading; and never seeing anything.

(very angry)

Prof. Smart - I could be with a nice lady, in her room, enjoying myself, but I am here getting fed up. Thirty million devils take me to the sky of purity if I hold this pen once more before having... Yes, yes, before having several girls with whom I could enjoy myself spending the hours nicely, without worrying, having as much pleasure as I want.

(more angry)

Prof. Smart - God damn, God damn, Hell and damnation. I live like an ass just working! Working! Always fed up and never enjoying! Definetely I'm fed up! Fed up! and don't want any more! Never more! Never more! Never more! I've said it, said it already and I will succeed! Succeed! Yes! Yes! It is said! Written here.

(putting his hand on his forehead)

Prof. Smart - It is done; in the bottom of my heart

(puts his hand on his heart)

Prof. Smart - I will therefore get dressed and go out for some fun; and finish my useful work!

(walks from one side to the other scratching his head, and growls, chews tobacco and sniffs snuff; goes out of the living room to the bed room; gets dress himself; and go out like a juggler preparing some trick. He speaks only when he reappears on the stage)

Prof. Smart - I was already anxious with so much writing and not seeing the person who, yesterday, sent to me the most affectionate words. 
(Black out)

\section{FIRST ACT}

\section{First Scene}

(Professor Smart is standing up over a table where a young woman is seated.Another young woman is seated over the table. Professor Smart is behind the former watching her write something. There is a lamp alight just abover her. Professor Smart takes the pupil's hand) ${ }^{6}$

Prof. Smart - My girl, hold this pen better; You, Madam, are not well seated.

(raising her body up)

Prof. Smart - Sit properly and keep your arm up.

(taking her arms)

Prof. Smart -This letter is not well done; better like that; this line is too thick; get some ink; Oh!, now yes, you got a wonderful picture; yes! Now you are really improving.

Mancilia - She always had a very good memory: yes, she is very sweet. Then, she will always make improvements; that's how it has been.

Prof. Smart - She has, however, my darling cousin, a great defect. Do you know what our cousin's defect is?

(Mancilia who was seating on the corner of the table, stands up smiling)

Mancília - What is it?

Prof. Smart - But don't you know yet?

Mancília - No.

Prof. Smart - It's being so beautiful, friendly and adorable, more being very, but very pleasing .

(the two young women smile. Mancilia laughs loudly)

6 A rubrica que abre a peça foi aqui adaptada considerando o enxerto da primeira cena de "Relacões Naturais". No original a rubrica é ; "Aparecem duas moças, uma assentada sobre uma mesa, outra em uma cadeira; uma luz em cima daquela; e um moço em pé, ensinando a que está assentada. Chamaremos a uma Mancília, à outra - Esterquilínia; ao moco, Espertalínio". (Qorpo Santo, op.cit. p.355) 
Esterquilínia - With a teacher like this one, even if I didn't want to I would learn very, very well.

Prof. Smart - I appreciate, and to such an extent that you have learned a lot; and very well. However it seems to me foolish to come here every day, or even at night to teach you, when I could do it in our house.

(The two young woman roar with laughter)

Esterquilínia - I have to admit... Each time he looks better: is it difficult to find as much as he offers and of such quality?

(Mancilia pretends to be in a pain)

Mancília - I'm a bit uncomfortable today. I feel my brain in a whirl... I don't know what's going on in my mind, what I have, who I am. ${ }^{7}$

Esterquilínia - What you have I know

(Shaking her head)

Esterquilínia - Yes...I know really.

Prof. Smart - It is something suitable for girls who study: Sometimes they are very clever: other times they are a bit confused or obtuse. Some days the memory of their lovers makess them contemplative, and other days, seeing their men makes them happy! And so that's how they live, and since they live with and for their lovers they start thinking like them, and write well, and tell ! what all of which is, really, quite remarkable.

Esterquilínia - That's right! That's right! It is just like that.

(pointing to Mancília)

Esterquilínia - I who know her very well, and very intimately, I can say. It's like that, my dear little cousin (showing five fingers of one hand): She has five boy friends.

Prof. Smart - Well! But five! It's a lot! Aren't you satisfied with two or three, that is the nice girls average? Godness! They are so many!

Mancília - Don't worry cousin, she is joking, I don't have more than one: and even he

7 Aqui acrescentou-se à pergunta da personagem "What I have?" uma nova pergunta, "Who am I para enfatizar a inconsistência psicológica da personagem e fortalecer o efeito cômico. 
is so far away...probably away at sea.

Prof. Smart - An do you love him so much?

Mancília - No; he is my cousin and that's why I care for him; Do I love him? Maybe... (She takes a breath)

Mancília - But I don't know, I really don't know. This heart of mine... I don't know what's wrong; it feels, it is in pain...

(She leaves the pen with which she has been writing and raises her head)

Mancília - I dreamt that my father had lived in the house of a relative and friend for a whole year; that there he fed himself; that the strength he got there produced me; that I belong or should belong to a son of this man...

(cleans her eyes)

Mancilia - That I'm one of the living rooms of his house; that...

(she falls over one of her hands)

Professor Smart - If I could, my dear student, alleviate you from the sorrow you feel in your heart; If I could console you for the affliction which it seems is reigning in your soul; If I were only strong enough to...

(He embraces her, and kisses her, or, at least, acts as if he had kissed her)

Professor Smart - Oh! What sweetness I would feel...I would enjoy the tranquility which I deserve, need and desired so much.

Esterquilínia - Very well; very well!

(She gets up from the table and goes out. Mancilia stands up and stretches)

Mancília - Oh! What pleasure I feel in this heart of mine, in this soul, it seems impossible that in paradise which was said to be so full of pleasure there could be greater delights in this world we live in ! May god prolongue my existence for a couple of centuries!

(embrancing Professor Smart)

Mancília - My darling, my friend! I am yours, you will be mine! A while a go you 
touched me in a way that almost killed me with pleasure! Yes! We will live together, and forever!

(Professor Smart stteping back)

Prof. Smart - Yes, Mancília, I love you too; I adore you...I want you; I desire you and my love is not like these modern poetic loves which are written on a piece of paper but soon disappear from the imagination and from the hearts of those who swear their dedication it to their lovers! It is big and long, it is strong; and so dense that there is no cloud in heaven, whose compactness could be compared with its density.

(An old woman enters)

Rocalipsa - What is it? My God! These girls. Cousin Joe, cousin Joe!

(putting her hands on her forehead)

Rocalipsa - Oh! It isn't the little cousin; it is Professor Smart. I thought it was he... This world is always like that. We are always making mistakes! Jesus; Holy Mary save these girls!

Mancília - What is it, Grandma? Grandmother I was dreaming, sleeping,

(the Grandaughter puts her hands on the Grandmother shoulders)

Mancília - So nicely, so sweetly. I was so satisfied. If you Grandma could imagine how nice my dream was! Look Grandma, I was with the dearest darling among my lovers. He was on top of my arms, and in the most pleasurable bed. The mattress, Grandma, wasn't made of hair, nor of feathers: Do you know Grandma what was it made of? It was made of something softer than satin; it was a kind of net...no, it was a kind of silk thread line; it was very dense, of the mildest stuff anyone could imagine; it was like those mist which in the winter emerge from the waters. And do you know who I was with? Who I was kissing, and touching! Who I was having ecstasy with? with...

(falls into Professor Smart's arms)

Mancília - With my... with the god of women; with my chosen above all, and finally favourite, my darling... I don't have to say more! My grandmother approves, doesn't she?

(putting her Grandmother's back)

Mancília - Yes, she does; I know she approves! 
(Rocalipsa, the Grandmother bends right over the body)

Rocalipsa - What could I do? You want each other; love each other; like each other... There is no choice other than to agree with you; You can be sure of my support.You must now deal with the bridal arrangements because these kind of things don't last long! Said and done is better than the best pie! Talking and delaying is worse than the worst bite!

Professor Smart - See Mancília, your Grandma loves us so much that today she became a poet. Don't you think?

Mancília - She always has been...but she used to hide her abilities; and just in a few happy moments she reveals it.

Prof. Smart - You know who will be upset for a while with what happened? And with our getting together; it is that old Adam who could have died of passion for you!

Mancilia - Don't worry! I already told him that he is too old and that he won't do for me; it looks like he accepted because he left and never came back.

Prof. Smart - These things bring back memories: longings...

Mancília - It didn't really matter: I would always be his darling! He, a man who, long ago, I rejected.

Rocalipsa - Be happy girls! Be happy! I will see my ancient old partner, see how he is doing with his erysipelas; his headaches, his stomach migraines, and with everything else he's used to suffering; because young oldmen, in other words, fresh husbands, aren't easy for me to find. Good by, so long!

(Rocalipsa emotionally embraces Professor Smart and Mancília. A girl gets in)

Isolina - Ai! you two?! What are you doing here?

Mancília - Go over there and find Grandmother, girl...

Isolina - I won't: I want to see what you are doing. I want to learn to be wise.

Mancília - You are too little to be wise. You are just a few years old; so,you can't learn anything from us.

Isolina - It doesn't matter. I watch now; learn; and when I am big I'll do it.

(Mancilia and Professor Smart roar with laughter) 
Prof. Smart - What do you think of this girl? How alive she is. How spirited, what a resourcefulness, and how elegant she is.

Mancília - It's true. There aren't many like her! The most interesting thing is her wish to learn now for when she is grown up.

Prof. Smart - And she's a good thinker.

Isolina - As we used to learn how to read, to write, to add, to weave, to embroider, to pick, when we are little we must learn everything else for when we are big to know what to do!

Mancília - The only way to escape from her is to go in to that room and tell her to take a flower to my grandmother.

(takes a flower from a vase)

Mancília - Take it Isolina. Take it to Grandmam. Tell her that this dahlia is one of the finest among ones they sent me from the Duke of Triumph's garden.

(the girl takes it and starts playing with the flower. Mancilia gives her arms to Professor Smart)

Mancília - Let's go, come on in.

(they leave the stage)

Isolina - They think I'm a fool!

(shaking her head and pointing to the place where they went)

Isolina - I know very well what they went in there for...They went to get married! They thought they would trick me... Well! I will not carry the flower while they stay there.

(walking and singing)

Isolina - I am a little girl, very nice. Someday I will marry...

(lifting the flower)

Isolina - to this smal flower! I don't want a wonderful boy because he would be impertinent and too insane. I prefer this flower because it doesn't hurt; it just gives pleasure to those who know how to look at.. 
(repeats the last speach and goes out. Black out)

\section{SECOND ACT}

\section{First Scene}

(Two billiard-tables on the stage, each one with its player - Peter and Paul. They play some shots before they start talking )

Peter - There isn't a better way to play! I've been trying to pot a ball for the last two hours to pocket a ball! And the other player isn't bothered! What do you Think, friend? That's how it is, isn't it?

Paul - Definitely! I have been having a lot of fun too. The best thing about it is that we never lose.

Peter - Oh ! that's nothing compared with the fact that the players avoid beating one another... That's something which I really appreciate!

(a ball jumps off the table)

Peter - If that had hit someone it would had gone through their stomach! What do you say Paul?

Paul - It is true; We are contenders without opponents...

(another ball jumps off)

Paul - Oh! that was an outstanding shot! But what is really important it is that the ball found a strong wall. Bigger than the walls of Sebastopol; I'm not comparing it with the great wall of China because it seems to be inferior! I am tired.

(Hiting another ball out with the stick! Professor Smart and Mancilia come back on he stage)

Mancília - How does it look to you, Smart? During our nuptials they transformed our living room into a snooker club! And it had to be just this game which I hate because of the ball's noise. Oh! what's so funny about that?

(takes a stick and throws it at a ball)

Prof. Smart - That's right. There is nothing funny about that! Now I can breathe! Gentlemen, who authorized you to come in here with these tables and play? 
Peter - We don't know! We just got in... we were around saw these tables here and got in. We were having a lot of fun! And you sir, What do you want now?

Mancília - I want you to take all this shit out imediately!

(taking a stick and turning it in the air, almost hitting one of the players. He jumps high)

Paul - Ai! You almost killed me.

(Peter for the public)

Peter - Before they hit me with another I'm getting out.

(he jumps two or three times and goes out)

Paul - It is better to be prudent! And when we are happy is a good time to leave.

(goes out doing pirouettes and with the arms open. Mancilia gets very angry)

Mancília - It was fine that they went home, otherwise I would have cut off their arms to make sticks and their heads to use as balls. With the legs I would make tables and with the skins table cloths.

(shouts from outside are heard. They are saying: Aux armes!, aux armes!)

Mancília - Are you listening Smart?! Listen? They are crying - Take up arms! Take up arms! What is it?

(very frightened)

Mancília - Hey? What am I hearing? What's happening little Smart?

Prof. Smart - It is true. What the hell is this ? Where could it be? Who could be suffering? Who would be the enemies? Oh! I'll see.

(Mancilia attacks Professor Smart)

Mancília - No! You won't leave me. I don't want you to leave. You couldn't go! I can't stay alone.

Prof. Smart - But if somebody is in pain, has been hit or is dying? Im going...

(trying to free himself from Mancilia's hands) 
Prof. Smart - I'm going, Mancília, going! I can't, do you understand, can't hear anybody groaning - crying, even talking and running - without helping.

(Mancilia cryes and is on her knees)

Mancília - And me? I'll die! die, die, if you leave me alone. No, you will not leave this place by the wounds of our lord Jesus Cristh! Little Smart, don't leave me! We got married yesterday and today am I already losing you!? And if they kill you! burn you! shoot you! What would become of me !? What would become of our little son, that's coming!? No! No!

(Mancilia grasps Professor Smart by the legs and stand up)

Mancília - You won't. Yes , will not go!

(Mancilia now speaks very sweetly)

Mancília - I know - you will listen to me. You won't leave me alone; and specially when there is danger, you won't leave me!

(They embrace each other and kiss)

Mancília - Let's enjoy ourselves on these tables they put in here...

(when they take the sticks, some soldiers with swords raised enter shoting)

Soldiers - You are under arrest! By the order of his excellency, most illustrous, wise, worthy, distinguished and eminent his honnour the Chief of Police of this most loyal city, and valorous consort of his Imperial Majesty of Brazil, Mr. Don Quixote of the Tulheries!

(Professor Smart talks to Mancília)

Prof. Smart - Run to the room while I deal with them!

(Mancilia absconding carefully with her eyes wide open and speaking to the public)

Mancília - Eh, eh...eh...Eh! Things aren't well at all! I can feel that something is going wrong.

(Professor Smart talks to the soldiers very frightened)

Prof. Smart - You Gentlemen are very wrong! The warrant isn't for this house, Gentlemen. There are not revolutionaries here! Don't you see? It's Just me, alone and... 
Soldier - I have already said - you are under arrest! You were accused of stillen these billiard-tables today! Yesterday you have stolen one young girl! and now you will be prosecuted for not having stolen two or threee instead of just one! What a fool! You are an ass! Now you, lazy bastard, you will rest just with that witch of yours!

(to the public)

Soldier - He ordered her to go to the room thinking someone here wanted her. What a damm fool!

(To Professor Smart and taking him by the arms)

Soldier - Come on! Come on I must go. You want, yes you want. It doesn't matter whether it will be easy, or foolish, or greasy! Are you listening to me? Now you Know that it is like that!

(They arrest him and take him with them. Professor Smart resists and cry)

Prof. Smart - I am inocent! Inocent! Leave me alone!

(The soldier goes behind Professor Smart pushing him)

Soldier - Come on fool, you are going to eat something better now.

\section{Second Scene}

(Mancilia comes back with her hair in disaray, very sad and weeping)

Mancília - My god! What is it! What am I seeing! What kind of dark shadows are spinning in front of me ! What a horror! My husband arrested and lost! My mother buried yesterday! My father almost dying, rather than already dead! My grandmother at this time in the most horrible agony! Me alone... helpless! Without any support in the world.

(claps her hands and falls on her knees)

Mancília - God of mercy, let even the lightest vapours of your grace, descend upon me! Bless one of your most unhappy daughters! Take from her of at least one half of the weight which is Killing her! Which is opressing her!

(with one of her hands on her eyebrow and standing up quickly) 
Mancilia - Ai! What is this, my God! Weren't my moral suffering enough... (with the hand on the forehead)

Mancília - And yet this disgusting headache...I don't know how I can still be alive; how can I speak; How I think; How can I even talk still! Ah!

(trying to move, walking a few steps)

Mancília - What a horrible dream this world is. My consciousness opresses me! I feel remorse devouring my heart as if it were a beast! I see death in front of my eyes! I see everything! The sepulchre opened! A Knife over that table! My spilt blood runs in streams! I faint ! I fall! My husband! My dear husband! Never again will I see him! Lost! Lost! I...I that... I mean...No!...I...Where am I? What do I? I would have gone...but...God! God! God.

(she kicks the floor with one foot)

Mancília - Won't you help me? I'm worth nothing in this mess... Oh! So there is no supreme being! yes! no... I believed...but now...Can I believe still? I've flee...I go...

(lifts up her arms, runs to the door and meets the policeman who is coming to get her. Gives a cry of pain and falls as if death; the soldiers run away frightened; after some minutes a lieutenant comes in)

Lieutenant - What's this?! The hostess fallen here! Could she be drunk or sick? Let's pick her up.

(Getting her by the shoulders and standing her up. Mancilia acts as if awake from a sleep and speaks the final phrase with very sweet words full of pain )

Mancília - Mr...For whom is... Tell me: What was that? Have you seen my husband? You, who are you? Has he already been released? Is he still in jail?

Lieutenant - Aren't you the hostess here?

Mancília - Me? No sir; however, if you are hungry, thirsty...I'll be able, since you've been so kind, to satisfy your needs... to kill...

(pointing her breast)

Mancília - To kill the hunger you have. 
Lieutenant - Why not? I came in thinking it was an hotel...

(appealing snobish)

Lieutenant - Being or not being...

(relaxing and sitting)

Lieutenant - Since you offer, I'll will eat here

(straighting the legs)

Lieutenant - And also...

(crossing the legs and supporting the head with one of the hands)

Lieutenant - And will also sleep! And something more I can do since the lady doesn't mind!

(To the public)

Lieutenant - She is so beautiful! very beautiful! So if she wanted to forget her husband, mainly now that I am very married- single, very single!

(singing)

Lieutenant - Which is better than married, very married, very married; very married;ve-ry- ma-rried! ve-ry-ma-rried! ve-ry-ma-rried! yeah! yeah, yeah,yeah,yeah,yeah.

\section{Third Scene}

(Professor Smart coming in)

Prof. Smart - Oh! You sir here!

Lieutenant - I beg your pardon, your Excellency knows me?

Prof. Smart - Yesterday when I saw you here I didn't recognize you, but after I remembered that, a long time ago, in the countryside, I met somebody who resembles you.

Lieutenant - It is true that I lived in a village in the country, in Cacapava; and you sir. Who are you? 
Prof. Smart - I am Professor Smart from Pigshire Cavalcante and Melro; and there I have a brother whose name is Misanthropo Mosquitos Rabiscaio.

Lieutenant - It is unbelievable; with this name the only one who lives there has the family name Carnaúba: his Excellency has the nick name of...

Prof. Smart - So, you must know that it is like that!

Lieutenant - I'm not sure yet...I know he has a brother; what is his christian name?

Prof. Smart - Joseph.

Lieutenant - Then it is true... But this Joseph.

(startled, opening his hands and eyes wide)

Lieutenant - He was fat, or strong; and you Sir look like a skeleton!

Prof. Smart - Don't be astonished; since I have been suffering so much in my life, the innumerable evils of which I have been a victim of - they were enough to kill me, but I'm still alive! I also feel that you no longer have the baby face you had when I last saw you; no longer that soft skined Adonis of... of satin or velvelt, of rose cheeks and thick lips! It is now an Adonis returned from the war, a little ruffer in his wrinkled face; the gestures, the manners! That's what the work and the years do to you. I remember, also your little wife, and your no less than five little sons, perhaps...

Lieutenant - I recognize that you know me. In fact I had some business at that time with a friend and partner of yours.

Prof. Smart - It is true that I was engaged to do some debt-collecting for a commercial house of a friend with whom I had intended to be a partner. Actually, it never came about. Are you planning to stay here very long?

Lieutenant - I am working for his Excellency the President of this province.

Prof. Smart - Have you come on important business?

Lieutenant - No.

Prof. Smart - Are you intending to continue coming here to this house?

Lieutenant - I came in thinking it was an hotel. 
Prof. Smart - It isn't, but you can come here to dinner any time you want.

Lieutenant - I'm very glad, very glad really...I have to walk around a bit, so I'm leaving.

Prof. Smart - I also have a lot of things to do.

(They say good bye to one another; Mancilia, or the wife of Professor Smart, gets in; when she sees him, she runs and shouts)

Mancília - My dear husband!

(she embraces him)

Mancília - How terrible your absence was for me!

Prof. Smart - Yes darling, darling Mancília, I have suffered the greatest torments during our cruel absence, altough everytime I...almost, almost died of missing you! Thank God we are, happily, joint again.

(Pause)

Prof. Smart - Who was a certain official which I met here, my friend?

Mancília - I don't know him; it was the first time I saw him; however he was very helpful: he lifted me in his arms since, with you so far away, I collapsed.

Prof. Smart - But I kept bothering him. I felt he was so impertinent!...

Mancília - I heard everything: He didn't want to trust you, or even believe you; But you have talked so well about the miracles which, many times, make the supreme being be shown to us; you argued that it was a miracle one man who have written more than two hundred books alone, as it was the appeareance of a great poet without art studies - or of a politician without scientific studies, and like that about all the great, marvellous truths, as about why he enjoys, sometimes, bringing light to us! I also liked very much hearing you talk on the subject of portraits...I mean - the inner self knowledge, through the external forms; through the facial features have you known the morals of your female friends ! One serious, angry and beautiful, or with an over light heart, or magnanimous; other, with an expression of a young friend - of joking, mocking, gay or cunning - but also full of noble feelings!

Prof. Smart - It's true, my dear friend; I described them as I understand them; they are like that! But do you know what I didn't like? It was when he said he liked to see the women cleansed and to go on... 
(takes her breasts, kiss her, and try to pull up her skirt, not too much)

Prof. Smart - Doing like that

(with gaiety)

Prof. Smart - As we used to do...

(Kissing her, taking her breasts and pulling up her starched skirt, etc.)

Prof. Smart - You know, don't you?

Mancília - So, has he really told you this? He is a thief! He robs your tastes and habits! Don't talk with him anymore, my friend; otherwise you will end up with nothing.

Prof. Smart - Oh! That's good! Then, just because he likes I already lose everything; no?! Don't let him bully you... and everything else is my business.

(Mancilia is surprised)

Mancília - With me? Don't think so badly about me if you don't want to see me transfixed with pain.

(Professor Smart to the public)

Prof. Smart - So that's what all women say - some die of legs others die of belts others I don't know of what - when their husbands remember some of their manias... No - some of their unsickness! Altough, each of them, put such a horn on their husbands! (opening the arms, comparing)

Prof. Smart - Some of them bring on the head truly kegs of thirty gallons; or others... oak casks of one hundred gallons! When, however, they are near to their husbands... oh! they look like saints; they swear; curse; get angry, and pity those poor husbands who don't succeed in making them believe they trust them! They are able to poison the air which should refresh and feed them.

(to Mancília, embracing and kissing her)

Prof. Smart - It's time, my little dear, to have some food ourselves.

Mancília - What can I give you since I have nothing?

Prof. Smart - What? 
Mancília - Brides don't eat in any other place than in bed;

(given small jumps)

Mancilia - They don't drink except in bed.So let's go there and you will have a feast.

Prof. Smart - This one, now, it is the best

(looking at the public)

Prof. Smart - I'm happy. I don't have to spend money or work: I drink the woman in bed; dress also the woman in bed, enjoy the woman in bed.

(dancing)

Prof. Smart - I am very happy! I'm very happy! very happy! and very happy!

(to the woman)

Prof. Smart - Won't you come with me?

Mancília - Why not!

(takes his hand, dances and sings with him)

Both - Who ever fell jealous - please - do the same! Do the same! Instead of spending - it is better to marry.

(these words can be repeated; and like that, it seems, it must finish the comedy)

Mancília - About the two white sheets,me, I have already, one which was written on today; the other my little old man (patting his shoulders) Mancília - Will write on tomorrow.

\section{By José Joaquim de Campos Leão \\ Qorpo-Santo}

Porto Alegre, may 5th,1866. Brasil. 


\section{Referências}

GRAVER, David. The Aesthetics of Disturbance: Anti-Art in Avant-Garde Drama. Theater: Theory/Text/Performance Series. Ann Arbor: The University of Michigan Press, 1995.

QORPO-SANTO, José Joaquim de Campos Leão. Teatro Completo. Rio de Janeiro: MEC/SMT/FUNARTE, 1980.

MOREIRA, Inês Cardoso Martins. "Ver-ouvir Stein." Inimigo Rumor (Rio de Janeiro), Rio de Janeiro / São Paulo, v. no17, p. 55-69, 2005. Disponível em http://revistamododeusar.blogspot.com/2012/11/gertrude-stein-ciclo-critico-ver-ouvir.html. Acesso em 3.8.19.

RYAN, Betsy A. Gertrude Stein's Theatre of the Absolute. UMI Research Press, Ann Arbour, Michigan, 1984.

STEIN, Gertrude. Opera \& Plays, ed. James Mellow. New York: Station Hill Press, 1987.

STEIN, Gertrude. Porta-retratos. Trad. Augusto de Campos, Florianópolis: Noa Noa, 1989.

STEIN, Gertrude. "Vozes de mulheres", "A seguir. Vida e cartas de Marcel Duchamp", "Capitão Walter Arnold", "Quero que isto seja uma peça. Uma peça”, "O Rei ou alguma coisa (convida-se o público a dançar)". Trad. Júlio Castañon Guimarães. O Percevejo: revista de Teatro, Crítica e Estética. Ano 8. N. 9. 2000. (Departamento de Teoria do Teatro. Programa de Pós-Graduação em Teatro. UNIRIO).

STEIN, Gertrude. "Contando os vestidos dela." Trad. Inês Cardoso Martins Moreira. Rio de Janeiro: Viveiros de Castro, 2001.

STEIN, Gertrude. "Parte IV - A questão da identidade - Uma Peça." Trad. Inês Cardoso Martins Moreira. Revista Inimigo Rumor. n. 17. Rio de Janeiro / São Paulo: Viveiros de Castro; CosacNaify, 2005 http://revistamododeusar.blogspot.com/2012/11/gertrude-stein-ciclo-critico-ver-ouvir.html. Acesso em 3.8.19.

STEIN, Gertrude. "Uma peça". "Três irmãs que não são irmãs". Trad. Inês Cardoso Martins Moreira. Revista OuvirOuver. V.6. N.2. p. 374- 391.Uberlândia: EDUFU, 2010. Disponível em http://www.seer.ufu.br/index.php/ouvirouver/issue/view/647. Acesso em 3.8.19.

STEIN, Gertrude. O que Você está Olhando - Teatro (1913-1920), Org.;Trad. Dirce Waltrick do Amarante e Luci Collin. São Paulo: Iluminuras, 2014. 
STEIN, Gertrude. "Doutor Faustus liga a luz." Trad. Fábio Fonseca de Melo. SãoPaulo: ConeSul, s/d.

SZONDI, Peter, Theory of Modern Drama. Cambridge: Polity Press, 1987.

WILLIAMS, Raymond. Drama em cena. Trad. Rogério Bettoni. São Paulo: CosacNaify, 2010.

Recebido em: 21/06/2019

Aprovado em: 10/07/2019 